\title{
Self-similar Sets as Hyperbolic Boundaries
}

KA-SING LAU \& XIANG-YANG WANG

\begin{abstract}
We show that, for an iterated function system $\left\{S_{j}\right\}_{j=1}^{N}$ of similitudes that satisfies the open set condition, there is a natural graph structure in the representing symbolic space to make it a hyperbolic graph, and the hyperbolic boundary is homeomorphic to the self-similar set generated by $\left\{S_{j}\right\}_{j=1}^{N}$.
\end{abstract}

\section{INTRODUCTION}

The theory of random walks on graphs has generated a lot of interest in probability, geometry, potential theory and harmonic analysis. Recently Denker and Sato [6-8] carried out an interesting study of this on the Sierpinski gasket $K$. They introduced a certain transition probability on the representing symbolic space and showed that the Martin boundary associated with this random walk is homeomorphic to the gasket $K$. Furthermore, in [8] they identified a subclass of harmonic functions from such a Markov chain with Kigami's harmonic functions on the Sierpinski gasket $[15,16]$. This provides a close link of the boundary theory with the current development of analysis on fractals.

In regard to the consideration in [6], Kaimanovich [14] introduced a hyperbolic structure ("augmented" tree) on the symbolic space of the Sierpinski gasket, and showed that the gasket can be identified naturally as the boundary of a hyperbolic graph. He further suggested that the Martin boundary in [6] can be obtained using the random walk technique in hyperbolic graphs ([1], [24]), and that this approach might also work for more general self-similar sets. As a first step to this investigation, we show in this paper that indeed certain self-similar sets can be given the hyperbolic structure through the augmented tree, and the set can be identified with the hyperbolic boundary of the tree.

Recall that for an iterated function system (IFS) of contractive maps $\left\{S_{j}\right\}_{j=1}^{N}$ on $\mathbb{R}^{d}$, there exists a unique non-empty compact subset such that $K=\bigcup_{j=1}^{N} S_{j}(K)$ 
([9], [12]). We call such $K$ an invariant set or attractor of the IFS. If in addition the contractive maps are similitudes, then $K$ is called a self-similar set. The IFS is said to satisfy the open set condition if there exists an bounded nonempty open set $V$ such that $S_{j}(V) \subset V$ and $S_{i}(V) \cap S_{j}(V) \neq \varnothing$ for $i \neq j$.

For each integer $n \geq 0$, we let $\Sigma_{n}=\{1, \ldots, N\}^{n}$ (convention $\Sigma_{0}=\varnothing$ ). $\Sigma=\bigcup_{n=0}^{\infty} \Sigma_{n}$ be the set of all finite indices, and let $\Sigma_{\infty}$ be the set of all infinite indices. For $\mathbf{i}=i_{1} \ldots i_{n}, \mathbf{j}=j_{1} \ldots j_{k} \in \Sigma$, denote $\mathbf{i} \mathbf{j}=i_{1} \ldots i_{n} j_{1} \ldots j_{k}$ be the concatenation; we also denote $\mathbf{v}_{1} \mathbf{v}_{2} \ldots \mathbf{v}_{k}$ by $\mathbf{v}^{k}$ if $\mathbf{v}_{i}=\mathbf{v}$ for all $i$. To deal with the different contraction ratios of $S_{i}$ 's, usually we group together the maps that have approximately equal contraction ratios: let $r_{j}$ be the contraction ratio of $S_{j}$ and let $r=\min \left\{r_{j} \mid 1 \leq j \leq N\right\}$,

$$
\mathcal{J}_{n}=\left\{\mathbf{j}=j_{1} \ldots j_{k} \in \Sigma \mid r_{j_{1}} \ldots r_{j_{k}} \leq r^{n}<r_{j_{1}} \ldots r_{j_{k-1}}\right\} .
$$

Note that for $m \neq n, \mathcal{J}_{m} \cap \mathcal{J}_{n}=\varnothing$, let $X=\bigcup_{n=0}^{\infty} \mathcal{J}_{n}$, where $\mathcal{J}_{0}=\{\varnothing\}$. In the case all $r_{i}, i=1, \ldots, n$ are equal, then $\mathcal{J}_{n}=\Sigma_{n}$ and $X=\Sigma$. In general $X$ is a proper subset of $\Sigma$. For example, if we let $\left\{S_{j}\right\}_{j=1}^{2}$ and $r_{1}=r_{2}^{2}$, then

$$
\mathbf{u}=\underbrace{22 \ldots 2}_{2 k+1} \notin \mathcal{J}_{n}, \quad \text { for any } n .
$$

Note that for each $\mathbf{i} \in \mathcal{J}_{n}, n \geq 1$, there is a unique $\mathbf{j} \in \mathcal{J}_{n-1}$ and $\mathbf{k} \in \Sigma$ such that $\mathbf{i}=\mathbf{j k}$. This defines a natural rooted tree structure on $X: \mathbf{i}, \mathbf{j}$ are said to be connected by a vertical edge and is denoted by $\mathbf{i} \sim \mathbf{j}$ if $\mathbf{i} \in \mathcal{J}_{n}, \mathbf{j} \in \mathcal{J}_{n-1}$ (or $\mathbf{j} \in \mathcal{J}_{n}$, $\left.\mathbf{i} \in \mathcal{J}_{n-1}\right)$ are related as the above. Let $\mathcal{E}_{v}$ be the set of all vertical edges, then $\left(X, \mathcal{E}_{v}\right)$ is a rooted tree with $o=\varnothing$ as the root. We also define a set of horizontal edges $\mathcal{E}_{h}$ as follow: for each $n$ and for $\mathbf{i}, \mathbf{j} \in \mathcal{J}_{n}, \mathbf{i} \sim \mathbf{j}$ if $S_{\mathbf{i}}(K) \cap S_{\mathbf{j}}(K) \neq \varnothing$ $\left(S_{\mathbf{j}}\right.$ means $\left.S_{j_{1}} \circ \cdots \circ S_{j_{k}}\right)$. We use $\mathcal{E}=\mathcal{E}_{v} \cup \mathcal{E}_{h}$ to denote the vertical and horizontal edges and $(X, \mathcal{E})$ the graph. This sets up an augmented rooted tree as in Kaimanovich [14].

We use the standard notation on hyperbolic graph $X$ introduced by Gromov ([10], [3], [24]). The hyperbolic boundary is defined as $\partial X=\hat{X} \backslash X$ where $\hat{X}$ is the compactification of $X$ under an ultra-metric $\rho_{a}(\cdot, \cdot)$ on $X$ (see Section 2). We prove the following results.

Theorem 1.1. Let $\left\{S_{j}\right\}_{j=1}^{N}$ be an IFS of contractive similitudes that satisfies the open set condition. Then $(X, \mathcal{E})$ is a hyperbolic graph.

Theorem 1.2. With the above assumptions, then the self-similar set $K$ is homeomorphic to the hyperbolic boundary of $(X, \mathcal{E})$. Furthermore, the Hölder equivalence holds if we assume some additional conditions on $\left\{S_{j}\right\}_{j=1}^{N}$ (condition $(\mathrm{H})$ in Section 4).

The open set condition in Theorem 1.1 is used to show that the lengths of horizontal geodesics are uniformly bounded (Lemma 3.1), which implies that the 
graph $(X, \mathcal{E})$ is hyperbolic (Theorem 2.3). For Theorem 1.2, we let $\Pi: \Sigma_{\infty} \rightarrow K$ be the map $\Pi(\mathbf{i})=\lim _{n \rightarrow \infty} S_{i_{1} \ldots i_{n}}\left(x_{0}\right)$. It is well known that the above limit exists and is independent of $x_{0} \in \mathbb{R}^{d}$. Let $\Sigma_{\infty} / \Pi$ denote the quotient space over the equivalent relation $\Pi(\mathbf{i})=\Pi(\mathbf{j})$. We show that $K$ and the hyperbolic boundary of $(X, \mathcal{E})$ are both homeomorphic to $\Sigma_{\infty} / \Pi$ and the theorem follows (Theorem 4.3, Proposition 4.4).

We remark that the horizontal edges can be defined more generally so that the hyperbolicity still holds (Definition 2.3, Proposition 3.4). The above consideration for self-similar sets can also be extended to the class of self-affine sets that are generated by the IFS of self-affine maps with equal contractive matrix $A$. This makes use of a special technique in [11] where the Euclidean distance in the key lemma (Lemma 3.1) is replaced by a pseudo-metric induced by $A$ (see Section 5).

In [1] (see also [24, p. 288]), Ancona proved that if $X$ is a hyperbolic graph and if there is a "reversible" transition probability on $X$, then by using a Harnacktype inequality, the associated Martin boundary is homeomorphic to the hyperbolic boundary of $X$. The transition probability in [6] is, however, not reversible and it is not direct to adopt the technique. We will consider this in a fore coming paper [13] with the setup on some post critically finite self-similar sets, which also satisfy the open set condition [5].

We organize the paper as following. In Section 2 we introduce some basic notation of the hyperbolic boundary, we also prove a criterion for an augmented rooted tree to be a hyperbolic graph. We prove Theorem 1.1 in Section 3 and Theorem 1.2 in Section 4. A consideration on the self-affine sets and some remarks on the case without the open set conditions are provided in Section 5.

\section{HyPERBOLIC GRAPHS}

Let $X$ be a countable infinite set, we say that $X$ is a graph if it is equipped with an adjacency relation (neighborhood) which is symmetrical, but non-reflexive; we use $x \sim y, x, y \in X$ to denote such relation. The degree of $x$ is the number of neighbors. In our consideration we will assume that $X$ is locally finite, i.e., the degree at each $x$ is finite. To visualize $X$ with the relation $\sim$, we draw a segment $[x, y]$ between the related vertices $x, y \in X$ and call it an edge; let $\mathcal{E}$ denote the set of edges on $X$ (note that $[x, x] \notin \mathcal{E}$ by the assumption on $\sim$ ). A (finite) path $p(x, y)$ from $x$ to $y$ is a sequence $\left[x_{1}, x_{2}, \ldots, x_{n}\right]$ with $x_{i} \sim x_{i+1}$ and $x=x_{1}$, $x_{n}=y$, we use $|p(x, y)|(=n-1)$ to denote the length of the path. We assume that every two points $x, y \in X$ are connected by a path. If such a path (with $x_{i} \neq x_{j}$ for all $\left.i, j\right)$ is unique, then we call it a tree.

A graph $X$ carries an integer-valued metric $d(x, y)$, which is the minimum among the lengths of all paths from $x$ to $y$. We denote the corresponding geodesic path by $\pi(x, y)$; note that such a path is not unique in general. Under this metric, $(X, d)$ is a proper metric space (every closed ball in $X$ is compact) and every two points can be connected by a geodesic. 
We choose an $o \in X$ as the origin and call it a root of the graph. We use $|x|$ to denote $d(o, x)$. For $x, y \in X$, we define the Gromov product ([3], [24]) of any two points $x, y \in X$ by

$$
|x \wedge y|=\frac{1}{2}(|x|+|y|-d(x, y)) .
$$

Definition 2.1. We say that $(X, \mathcal{E})$ is $\delta$-hyperbolic (with respect to $o$ ) if there exists $\delta \geq 0$ such that

$$
|x \wedge y| \geq \min \{|x \wedge z|,|z \wedge y|\}-\delta, \quad \forall x, y, z \in X .
$$

It is known that $X$ is $\delta$-hyperbolic with respect to a particular $o \in X$ if and only if it is $2 \delta$-hyperbolic for any fixed root $o^{\prime} \in X$ [3]. Hence hyperbolicity is independent of the choice of the root. In general $|x \wedge y|$ is roughly the distance from $o$ to $\pi(x, y)$ in the following sense:

$$
d(o, \pi(x, y))-2 \delta \leq|x \wedge y| \leq d(o, \pi(x, y)) .
$$

A geodesic triangle is a triangle consistsing of three points $x, y, z$ as vertices, together with the three geodesic $\operatorname{arcs} \pi(x, y), \pi(y, z), \pi(z, x)$ as sides; the triangle is called $\delta$-thin if every point on any one of the sides is at distance at most $\delta$ to one of the other two sides. There is a more geometric characterization of the $\delta$-hyperbolicity.

Proposition 2.1. A graph $(X, \mathcal{E})$ is $\delta$-hyperbolic for some $\delta>0$ if and only if there exists $\delta^{\prime}>0$ such that every geodesic triangle in $X$ is $\delta^{\prime}$-thin.

As in [24], we choose $a>0$ such that

$$
a^{\prime}=e^{3 \delta a}-1<\sqrt{2}-1
$$

and define for $x, y \in X$,

$$
\rho_{a}(x, y)= \begin{cases}\exp (-a|x \wedge y|), & x \neq y, \\ 0, & x=y .\end{cases}
$$

Then

$$
\rho_{a}(x, y) \leq\left(1+a^{\prime}\right) \max \left\{\rho_{a}(x, z), \rho_{a}(y, z)\right\}, \quad \forall x, y, z \in X .
$$

This means $\rho_{a}(\cdot, \cdot)$ is an ultra-metric. It is not a metric, but is equivalent to the following metric:

$$
\theta_{a}(x, y)=\inf \left\{\sum_{i=1}^{n} \rho_{a}\left(x_{i-1}, x_{i}\right) \mid n \geq 1, x=x_{0}, x_{1}, \ldots, x_{n}=y, x_{i} \in X\right\} .
$$


Since $\rho_{a}$ and $\theta_{a}$ define the same topology, in our consideration we will use $\rho_{a}$ instead of $\theta_{a}$ for simplicity. It is known that for any sequence $\left\{x_{n}\right\}_{n=1}^{\infty}$ such that $\lim _{n \rightarrow \infty}\left|x_{n}\right|=\infty$,

$\left\{x_{n}\right\}$ is Cauchy in the ultra-metric $\rho_{a}(x, y)$ if and only if $\lim _{m, n \rightarrow \infty}\left|x_{m} \wedge x_{n}\right|=\infty$.

Definition 2.2. Let $\hat{X}$ denote the completion of the graph $X$ under $\rho_{a}$; we call $\partial X=\hat{X} \backslash X$ the hyperbolic boundary of $X$.

The hyperbolic boundary $\partial X$ is a compact set. It is often useful to identify $\xi \in \partial X$ with the geodesic rays in $X$ that converge to $\xi$. (By a geodesic ray, we mean an infinite path $\pi\left[x_{1}, x_{2}, \ldots\right]$ such that $x_{i} \sim x_{i+1}$ and any finite segment of the path is a geodesic.) Note that two geodesic rays $\xi=\left[x_{1}, x_{2}, \ldots\right]$ and $\eta=\left[y_{1}, y_{2}, \ldots\right]$ with $\left|x_{1}\right|=\left|y_{1}\right|$ are equivalent as Cauchy sequence if and only if

$$
d\left(x_{n}, y_{n}\right) \leq c \delta
$$

for all but finitely many $n$, where $c>0$ is independent of the rays [24].

In order to consider the self-similar sets, we need some further notation of a graph. Let $X$ be a tree with a root $o$, we denote its edges by $\mathcal{E}_{v}$ and refer them as the vertical edges. We say that $x$ belongs to the $n$-th level if $d(o, x)=n$; we use $x^{[-k]}$ to denote the $k$-th ancestor of $x$, the unique point in $(n-k)$-th level such that $d\left(x^{-[k]}, x\right)=k$. Note that each $x$ can have multiple descendants but a unique ancestor on each level.

Definition 2.3 (Kaimanovich [14]). Let $X$ be a tree with a root $o$. We introduce a set of horizontal edges $\mathcal{E}_{h}$ in $X$ as follows: $\mathcal{E}_{h}$ is symmetrical but nonreflexive, and

$[x, y] \in \mathcal{E}_{h} \Rightarrow|x|=|y|, \quad$ and either $x^{[-1]}=y^{[-1]}$ or $\left[x^{[-1]}, y^{[-1]}\right] \in \mathcal{E}_{h}$.

Let $\mathcal{E}=\mathcal{E}_{v} \cup \mathcal{E}_{h}$ and call $(X, \mathcal{E})$ an augmented rooted tree.

It is obvious that $d\left(x^{[-1]}, y^{[-1]}\right) \leq d(x, y)$ for all $x, y \in X$. For any $x$, $y \in X$, we say that the geodesic $\pi(x, y)$ is an $h$-geodesic if the path consists of horizontal edges only, and a $v$-geodesic if the path consists of vertical edges only; it is called a canonical geodesic if there exist $u, v \in \pi(x, y)$ such that

(i) $\pi(x, y)=\pi(x, u) \cup \pi(u, v) \cup \pi(v, y)$ with $\pi(u, v)$ a horizontal path and $\pi(x, u), \pi(v, y)$ vertical paths;

(ii) for any geodesic path $\pi^{\prime}(x, y)$, dist $(o, \pi(x, y)) \leq \operatorname{dist}\left(o, \pi^{\prime}(x, y)\right)$.

Since a geodesic path may not be unique, condition (ii) is to require the horizontal part of the canonical geodesic to be on the highest level (see Figure 2.1). 


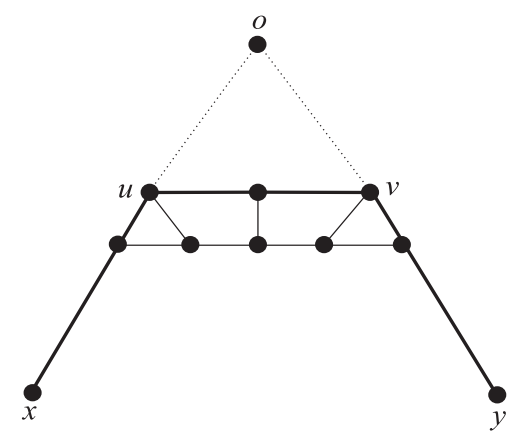

FIGURE 2.1. Canonical geodesic

Following [14], we can use the following moves repeatedly to change the geodesic without increasing the length: for $u, v \in \pi(x, y),|u|=|v|$,

$$
\left[u, v, v^{[-1]}\right] \rightarrow\left[u, u^{[-1]}, v^{[-1]}\right] \text { and }\left[u^{[-1]}, u, v\right] \rightarrow\left[u^{[-1]}, v^{[-1]}, v\right] .
$$

By using this, it is straightforward to check the following result.

Proposition 2.2. Any two points $x, y$ in an augmented rooted tree can be joined by a canonical geodesic $\pi(x, y)$. In this case $|x \wedge y|=\ell-h / 2$ where $\ell$ and $h$ are respectively the level and length of the horizontal part of the geodesic.

Our main theorem in this section is the following.

Theorem 2.3. An augmented rooted tree $X$ is hyperbolic if and only if there exists $k>0$ such that any $h$-geodesic is bounded by $k$.

Proof. We use the characterization of $\delta$-hyperbolicity in Proposition 2.1 to prove the necessity. Suppose all the geodesic triangles are $\delta$-thin, we show that any $h$-geodesic is bounded by $5 \delta$. If otherwise, let $x, y$ be such that $|x|=|y|$ and let $\pi(x, y)$ be an $h$-geodesic of length $>5 \delta$. Consider the geodesic triangle $o x y$. Let $u$ be a point in $\pi(x, y)$ such that $\pi(x, u), \pi(u, y)>2 \delta$ (e.g., the "mid-point"). By the $\delta$-hyperbolicity, there exists $v \in \pi(o, x)$ (or $v \in \pi(o, y)$ ) such that $d(u, v) \leq \delta$. Since $x, u$ are on the same horizontal level, we have $d(x, v)=|x|-|v|=|u|-|v| \leq \delta$ also. This implies that

$$
2 \delta<d(x, u) \leq d(x, v)+d(v, u) \leq 2 \delta,
$$

a contradiction.

To prove the sufficiency, let $k$ be the bound of the length of the $h$-geodesics. If $X$ is not $\delta$-hyperbolic for any $\delta>0$, by the contraposition of Definition 2.1, we can find $x, y, z$ such that

$$
|x \wedge y| \leq \min \{|x \wedge z|,|y \wedge z|\}-k
$$


By Proposition 2.2, we can assume the geodesics joining the pairs of $x, y, z$ are canonical. Let $\ell_{i}, i=1,2,3$ denote the lengths of the horizonal paths in $\pi(x, y), \pi(x, z)$ and $\pi(y, z)$ respectively. Also we let $h_{i}, i=1,2,3$ denote the corresponding levels of the horizontal paths. Using the notation above, we can rewrite $(2.5)$ as

$$
h_{1}-\ell_{1} / 2+k \leq \min \left\{h_{2}-\ell_{2} / 2, h_{3}-\ell_{3} / 2\right\} .
$$

Noting that $\ell_{i} \leq k,(i=1,2,3)$, we have $h_{1} \leq \min \left\{h_{2}, h_{3}\right\}$. Without loss of generality, we assume that $h_{2} \leq h_{3}$.

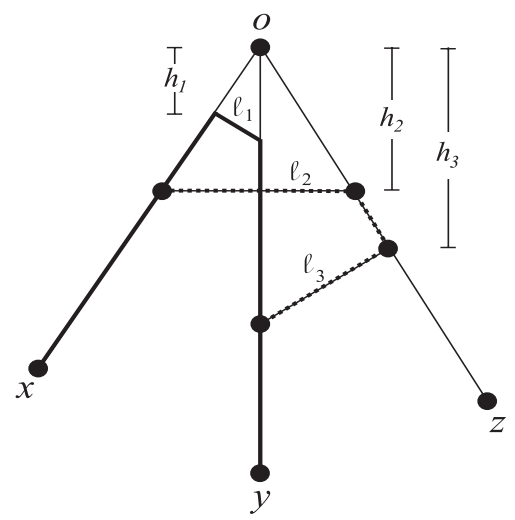

FIGURE 2.2. Geodesic segments

We now construct a new path $p(x, y)$ from $x$ to $y$ (see Figure 2.2): start form $x$, move along $o x$, change to the horizontal path of $\pi(x, z)$, then move on $o z$ and reach the horizontal path of $\pi(z, y)$, then follow this path to reach $y$. Since $\pi(x, y)$ is a geodesic, we have $|\pi(x, y)| \leq|p(x, y)|$. By comparing the difference in length of the two paths and making use of the property that a geodesic has minimum length, we have

$$
\left(h_{2}-h_{1}\right)+\ell_{1}+\left(h_{3}-h_{1}\right) \leq \ell_{2}+\left(h_{3}-h_{2}\right)+\ell_{3} .
$$

i.e.,

$$
2\left(h_{2}-h_{1}\right)+\ell_{1} \leq \ell_{2}+\ell_{3} .
$$

On the other hand, (2.6) implies that

$$
h_{1}-\ell_{1} / 2+k \leq h_{2}-\ell_{2} / 2 .
$$

It follows that

$$
2 k+\ell_{2} \leq 2\left(h_{2}-h_{1}\right)+\ell_{1} \leq \ell_{2}+\ell_{3} .
$$

We see that $\ell_{3} \geq 2 k$. It contradicts that all $h$-geodesics in $X$ are bounded by $k$. $\square$ 


\section{Self-Similar SeTS}

In the rest of the paper we assume that $\left\{S_{j}\right\}_{j=1}^{N}$ is an iterated function system (IFS) consisting of $N$ contractive similitudes on $\mathbb{R}^{d}$ :

$$
S_{j}(x)=r_{j} R_{j}\left(x+d_{j}\right), \quad j=1, \ldots, N,
$$

where $0<r_{j}<1$ is the contraction ratio of $S_{j}, R_{j}$ is an orthonormal $d \times d$ matrix and $d_{j} \in \mathbb{R}^{d}$. We use the notation defined in Section 1 .

We assume that the family $\left\{S_{j}\right\}_{j=1}^{N}$ satisfies the open set condition, and denote the self-similar set by $K$. For each $n$, the family $\left\{S_{\mathbf{u}} \mid \mathbf{u} \in \mathcal{J}_{n}\right\}$ has the OSC with the same open set $V$ as the original family $\left\{S_{j}\right\}_{j=1}^{N}$, and

$$
K=\bigcup\left\{S_{\mathbf{u}}(K) \mid \mathbf{u} \in \mathcal{J}_{n}\right\},
$$

where $\mathcal{J}_{n}$ is defined in (1.1).

Lemma 3.1. Suppose the IFS $\left\{S_{j}\right\}_{j=1}^{N}$ of similitudes satisfies the OSC. Then for any $a>0$, there exists $k^{\prime}>0$ (depending on a) such that for any $n$ and $D \subset \mathbb{R}^{d}$ with $\operatorname{diam}(D) \leq \operatorname{ar}^{n}\left(r=\min \left\{r_{1}, \ldots, r_{N}\right\}\right)$,

$$
\#\left\{\mathbf{u} \in \mathcal{J}_{n} \mid S_{\mathbf{u}}(K) \cap D \neq \varnothing\right\} \leq k^{\prime} .
$$

This is a consequence of [21, Proposition 2.1], for the IFS to satisfy the weak separation condition and hence the OSC. On the other hand, a straightforward proof is not difficult and we omit the details.

We now construct a graph on $X=\bigcup_{n=0}^{\infty} \mathcal{J}_{n}$. It is easy to see that for any integer $k<n$, and $\mathbf{u} \in \mathcal{J}_{n}$, there exists a unique $\mathbf{v} \in \mathcal{J}_{k}$ and $\mathbf{v}^{\prime} \in \Sigma$, such that $\mathbf{u}=\mathbf{V} \mathbf{v}^{\prime}$. We denote this unique $\mathbf{v}$ by $\mathbf{u}^{[-(n-k)]}$ and call it $(n-k)$-th ancestor of $\mathbf{u}$. The natural tree structure on $X$ is to take $\varnothing$ as the root $o$, and let $\mathcal{E}_{v}$ be the set of vertical edges defined by

$$
\mathcal{E}_{v}=\left\{\left(\mathbf{u}^{[-1]}, \mathbf{u}\right) \mid \mathbf{u} \in X \backslash\{\varnothing\}\right\} .
$$

We define another set of horizontal edges by

$$
\mathcal{F}_{h}=\left\{(\mathbf{u}, \mathbf{v}) \mid \exists n>0 \text { such that } \mathbf{u} \neq \mathbf{v} \in \mathcal{J}_{n} \text { and } K_{\mathbf{u}} \cap K_{\mathbf{v}} \neq \varnothing\right\} \text {, }
$$

where $K_{\mathbf{u}}:=S_{\mathbf{u}}(K)$. Let $\mathcal{E}=\mathcal{E}_{v} \cup \mathcal{E}_{h}$.

Theorem 3.2. Suppose the IFS $\left\{S_{j}\right\}_{j=1}^{N}$ of similitudes satisfies the OSC. Then the augmented rooted tree $(X, \mathcal{E})$ is hyperbolic. 
Proof. First we see that $\mathcal{E}$ is locally finite. It follows from Lemma 3.1 that there exists $k^{\prime}>0$ such that for any integer $n>0$ and $\mathbf{v} \in \mathcal{J}_{n}$,

$$
\#\left\{\mathbf{u} \in \mathcal{J}_{n} \mid K_{\mathbf{u}} \cap K_{\mathbf{v}} \neq \varnothing\right\} \leq k^{\prime} .
$$

Therefore $\mathbf{v}$ has at most $k^{\prime}$ neighbors in the same level, also it has one ancestor. On the other hand, let $\mathbf{u}$ be a descendant of $\mathbf{v}$, i.e., $\mathbf{u}^{[-1]}=\mathbf{v}$, then $K_{\mathbf{u}} \subset K_{\mathbf{v}}$, hence $K_{\mathbf{u}} \cap K_{\mathbf{v}} \neq \varnothing$. Let $D=K_{\mathbf{v}}$; by using Lemma 3.1 again, we have

$$
\#\left\{\mathbf{u} \in X \mid \mathbf{u}^{[-1]}=\mathbf{v}\right\} \leq k^{\prime \prime} .
$$

Hence for $\mathbf{v} \in X$,

$$
\operatorname{deg}(\mathbf{v})=\#\{(\mathbf{u}, \mathbf{v}) \in \mathcal{E}: \mathbf{u} \in X\} \leq k^{\prime}+k^{\prime \prime}+1,
$$

and $\mathcal{E}$ is locally finite.

Next we show that the lengths of the $h$-geodesics are bounded by some constant, then Theorem 2.3 implies that $(X, \mathcal{E})$ is hyperbolic. Suppose otherwise, for any integer $m>0$, there exists an $h$-geodesic $\pi\left(\mathbf{u}_{0}, \mathbf{u}_{3 m}\right)=\left[\mathbf{u}_{0}, \mathbf{u}_{1}, \ldots, \mathbf{u}_{3 m}\right]$ with $\mathbf{u}_{i} \in \mathcal{J}_{n}$. We consider the $m$-th ancestors $\mathbf{v}_{i}=\mathbf{u}_{i}^{[-m]}$ and the path $\left[\mathbf{v}_{0}, \ldots, \mathbf{v}_{3 m}\right]=\left[\mathbf{u}_{0}^{[-m]}, \ldots, \mathbf{u}_{3 m}^{[-m]}\right]$. Let

$$
p\left(\mathbf{v}_{0}, \mathbf{v}_{3 m}\right)=\left[\mathbf{v}_{i_{0}}, \ldots, \mathbf{v}_{i_{\ell}}\right], \quad \mathbf{v}_{i_{j}} \in\left\{\mathbf{v}_{0}, \ldots, \mathbf{v}_{3 m}\right\},
$$

be the shortest horizontal path connecting $\mathbf{v}_{0}$ and $\mathbf{v}_{3 m}$. By the geodesic property of $\pi\left(\mathbf{u}_{0}, \mathbf{u}_{3 m}\right)$, it is clear that

$$
\ell=\left|p\left(\mathbf{v}_{0}, \mathbf{v}_{3 m}\right)\right| \geq\left|\pi\left(\mathbf{u}_{0}, \mathbf{u}_{3 m}\right)\right|-2 m=m .
$$

Now choose $m \geq k^{\prime}$ such that $(3 m+1) r^{m} \leq 1$, where $k^{\prime}$ is as in Lemma 3.1. Let

$$
D=\bigcup_{i=0}^{3 m} K_{\mathbf{u}_{i}} .
$$

From $\operatorname{diam} K_{\mathbf{u}_{i}} \leq r^{n} \operatorname{diam}(K)(i=0,1, \ldots, 3 m)$, it is straightforward to show that

$$
\operatorname{diam}(D) \leq(3 m+1) r^{n} \operatorname{diam}(K) \leq r^{n-m} \operatorname{diam}(K) .
$$

Note that $K_{\mathbf{u}_{i}} \subset K_{\mathbf{v}_{i}}$, we see that $K_{\mathbf{v}_{i_{j}}} \cap D \neq \varnothing$ for each $j=0,1, \ldots, \ell$. It follows that

$$
\#\left\{\mathbf{v} \in \mathcal{J}_{n-m} \mid K_{\mathbf{v}} \cap D \neq \varnothing\right\} \geq \ell+1>m \geq k^{\prime} .
$$

It contradicts Lemma 3.1, and the proof is complete. 
It is easy to see that the unit interval $[0,1]$ associated with the IFS $S_{1}(x)=x / 2$, $S_{2}(x)=(x+1) / 2$, or the Sierpinski gasket associated with the maps $S_{i}(x)=$ $\left(x+p_{i}\right) / 2, i=1,2,3$, where $p_{1}, p_{2}, p_{3}$ are the vertices of an equilateral triangle, the corresponding augmented rooted trees are hyperbolic [14]. We remark that these examples can be put into another framework of sub-Markovian graphs introduced by Gromov ([10], [4]) also, which is analogous to the augmented rooted tree. However, that approach seems less straightforward, and it needs to use the property of subshift of finite type, which cannot cover all the IFS with the OSC considered here (see the example in Section 4).

Recently there is a class of self-similar sets introduced by Kigami $[15,16]$ that has received a lot of attention in the analysis of fractals: for the IFS $\left\{S_{j}\right\}_{j=1}^{N}$ of similitudes, let

$$
C_{K}=\bigcup\left\{S_{i}(K) \cap S_{j}(K) \mid i, j=1,2, \ldots, N, i \neq j\right\}, \quad C=\Pi^{-1}\left(C_{K}\right) ;
$$

we say that the IFS has the post critically finite (p.c.f) property, if the set $\mathcal{P}=$ $\bigcup_{n=1}^{\infty} \sigma^{n}(C)$ is finite, where $\sigma(\cdot)$ is the left shift operator on $\Sigma_{\infty}$, i.e., $\sigma\left(i_{1} i_{2} \ldots\right)=$ $i_{2} i_{3} \ldots$. The self-similar set generated by such IFS is finitely ramifiable, i.e., it becomes disconnected by removing a finite set points (for example, the Sierpinski gasket). In [5] it is proved that if the similitudes satisfies the p.c.f, and the contractions $\left\{r_{j} R_{j}\right\}_{j=1}^{N}$ are commensurable (i.e., there exists a matrix $A$ such that for each $j, r_{j} R_{j}=A^{n_{j}}$ for some positive integers $n_{j}$ ), then it has the OSC.

Corollary 3.3. Suppose the IFS $\left\{S_{j}\right\}_{j=1}^{N}$ of similitudes has the p.c.f property and the contraction ratios are commensurable, then the augmented rooted tree $(X, \mathcal{E})$ defined in Theorem 3.2 is hyperbolic.

In some cases it is also useful to define the horizontal paths in the augmented trees by another set of paths. For example, in the Sierpinski carpet, it is more natural to define the neighbors to be $K_{\mathbf{u}} \cap K_{\mathbf{v}}, \mathbf{u}, \mathbf{v} \in \Sigma_{n}$, to have Hausdorff dimension equals 1 .

Proposition 3.4. Suppose the IFS $\left\{S_{j}\right\}_{j=1}^{N}$ of similitudes satisfies the OSC. For $\alpha \geq 0$, define

$$
\mathcal{E}_{h}^{(\alpha)}=\bigcup_{n=1}^{\infty}\left\{(\mathbf{u}, \mathbf{v}) \mid \mathbf{u}, \mathbf{v} \in \mathcal{J}_{n}, \operatorname{dim}_{H}\left(K_{\mathbf{u}} \cap K_{\mathbf{v}}\right) \geq \alpha\right\}
$$

and let $\mathcal{E}^{(\alpha)}=\mathcal{F}_{h}^{(\alpha)} \cup \mathcal{F}_{v}$. Then $\left(X, \mathcal{E}^{(\alpha)}\right)$ is a hyperbolic graph.

Proof. The proof is the same as the above with some obvious modification. $\square$

4. Self-similar Set as Hyperbolic Boundary

We assume that $(X, \mathcal{E})$ is defined as in (3.2). It is easy to show that for any geodesic ray $\xi=\pi\left[\mathbf{u}_{1}, \mathbf{u}_{2}, \ldots\right]$, there exists $i_{1}, i_{2}, \ldots \in\{1, \ldots, N\}$ such that for 
each $k \geq 0, \mathbf{u}_{k}=i_{1} \ldots i_{n_{k}}$ for some integer $n_{k}$. Also each geodesic ray, up to equivalence, is in one-to-one correspondence with an element in the hyperbolic boundary $\partial X[24]$. When there is no confusion, we will identify the points in the hyperbolic boundary with equivalent classes of geodesic rays.

We define a map $\Phi$ from the set of all geodesic rays to the self-similar set $K$ by

$$
\Phi(\xi)=\lim _{n \rightarrow \infty} S_{\mathbf{u}_{n}}\left(x_{0}\right) .
$$

It is known that the above limit is independent of $x_{0} \in \mathbb{R}^{d}$. To justify that $\Phi$ is well defined, we prove the following result.

Lemma 4.1. If $\xi$ and $\eta$ are equivalent geodesic rays, then $\Phi(\xi)=\Phi(\eta)$.

Proof. Let $\xi=\pi\left[\mathbf{u}_{1}, \mathbf{u}_{2}, \ldots\right]$ and $\eta=\pi\left[\mathbf{v}_{1}, \mathbf{v}_{2}, \ldots\right]$. It follows from (2.4) that there exists $c>0$ such that

$$
d\left(\mathbf{v}_{n}, \mathbf{u}_{n}\right) \leq c \delta
$$

for all $n \geq 0$, where $\delta$ is the constant in the definition of a hyperbolic graph.

Let $\mathbf{u}_{n}=\mathbf{t}_{0}, \mathbf{t}_{1}, \ldots, \mathbf{t}_{k}=\mathbf{v}_{n}$ be a canonical geodesic from $\mathbf{u}_{n}$ to $\mathbf{v}_{n}$, then $k \leq c \delta$. The canonical geodesic can be written in three parts, two vertical and one horizontal parts: $\mathbf{t}_{0}, \ldots, \mathbf{t}_{i} ; \mathbf{t}_{i}, \ldots, \mathbf{t}_{j}$ and $\mathbf{t}_{j}, \ldots, \mathbf{t}_{k}$. For the horizontal part, we assume that $\mathbf{t}_{i}, \ldots, \mathbf{t}_{j} \in \mathcal{J}_{\ell_{n}}$. Note that

$$
K_{\mathbf{t}_{0}} \subset K_{\mathbf{t}_{1}} \subset \cdots \subset K_{\mathbf{t}_{i}} \quad \text { and } \quad K_{\mathbf{t}_{j}} \supset K_{\mathbf{t}_{j-1}} \supset \cdots \supset K_{\mathbf{t}_{k}} .
$$

Taking any $x_{0} \in K$, then for the initial and the tail part, we have

$$
\left|S_{\mathbf{t}_{0}}\left(x_{0}\right)-S_{\mathbf{t}_{i}}\left(x_{0}\right)\right| \leq \operatorname{diam}\left(K_{\mathbf{t}_{i}}\right)=r_{\mathbf{t}_{i}} \operatorname{diam}(K) \leq r^{\ell_{n}} \operatorname{diam}(K),
$$

where $r_{j_{1} \ldots j_{m}}:=r_{j_{1}} \ldots r_{j_{m}}$ for $j_{1} \ldots j_{m} \in \Sigma$. Similarly

$$
\left|S_{\mathbf{t}_{j}}\left(x_{0}\right)-S_{\mathbf{t}_{k}}\left(x_{0}\right)\right| \leq \operatorname{diam}\left(K_{\mathbf{t}_{j}}\right)=r_{\mathbf{t}_{j}} \operatorname{diam}(K) \leq r^{\ell_{n}} \operatorname{diam}(K) .
$$

For the intermediate part $\left[\mathbf{t}_{i}, \ldots, \mathbf{t}_{j}\right]$, they are in the same horizontal level $\ell_{n}$, hence $r_{\mathrm{t}_{i}}, \ldots, r_{\mathrm{t}_{j}} \leq r^{\ell_{n}}$ and $j-i \leq c \delta$. A simple argument shows that

$$
\left|S_{\mathbf{t}_{i}}\left(x_{0}\right)-S_{\mathbf{t}_{j}}\left(x_{0}\right)\right| \leq(j-i+1) r^{\ell_{n}} \operatorname{diam}(K) \leq(c \delta+1) r^{\ell_{n}} \operatorname{diam}(K) .
$$

Putting these together, we get

$$
\left|S_{\mathbf{v}_{n}}\left(x_{0}\right)-S_{\mathbf{u}_{n}}\left(x_{0}\right)\right| \leq C r^{\ell_{n}},
$$

for some constant $C>0$. Note that $\ell_{n}-c \delta \leq\left|\mathbf{u}_{n} \wedge \mathbf{v}_{n}\right| \leq \ell_{n}$ (see (2.1)), hence $\lim _{n \rightarrow \infty} \ell_{n}=\lim _{n \rightarrow \infty}\left|\mathbf{u}_{n} \wedge \mathbf{v}_{n}\right|=+\infty$. It follows that $\lim _{n \rightarrow \infty} S_{\mathbf{v}_{n}}\left(x_{0}\right)=$ $\lim _{n \rightarrow \infty} S_{\mathbf{u}_{n}}\left(x_{0}\right)$ and the lemma follows. 
Corollary 4.2. If $\xi=\pi\left[\mathbf{u}_{0}, \mathbf{u}_{1}, \ldots\right]$ and $\eta=\pi\left[\mathbf{v}_{0}, \mathbf{v}_{1}, \ldots\right]$ are equivalent geodesic rays starting from the root 0 , then there exists an integer $k$, such that

$$
\mathbf{u}_{i}=\mathbf{v}_{i}, i \leq k \quad \text { and } \quad d\left(\mathbf{u}_{i}, \mathbf{v}_{i}\right)=1, i>k .
$$

Proof. By Lemma 4.1, $\Phi(\xi)=\Phi(\eta)=x$ for some $x \in K$. This implies $x \in S_{\mathbf{u}_{n}}(K) \cap S_{\mathbf{v}_{n}}(K)$ for all $n=0,1, \ldots$, hence either $\mathbf{u}_{n}=\mathbf{v}_{n}$ or $d\left(\mathbf{u}_{n}, \mathbf{v}_{n}\right)=1$. To complete the proof, we need only see that if $d\left(\mathbf{u}_{n}, \mathbf{v}_{n}\right)=1$, then $d\left(\mathbf{u}_{n+k}, \mathbf{v}_{n+k}\right)=1$ for all $k>0$. This is trivial, since if there exists some $k>0$, such that $\mathbf{u}_{n+k}=\mathbf{v}_{n+k}$, say, it is the first one, then it has two one-step ancestor and it is impossible.

Theorem 4.3. Let $K$ be the self-similar set of the IFS $\left\{S_{j}\right\}_{j=1}^{N}$ which satisfies the OSC. Then $\Phi: \partial X \rightarrow K$ is a bijection and satisfies

$$
|\Phi(\xi)-\Phi(\eta)| \leq C \rho_{a}(\xi, \eta)^{\alpha}, \quad \forall \xi, \eta \in \partial X,
$$

where $\rho_{a}$ is the ultra-metric on $\partial X$ as in (2.2) and $\alpha=-\log r / a$. In this case $\partial X$ is homeomorphic to $K$.

Proof. For $x \in K$, there exists a sequence $i_{1} i_{2} \ldots \in \Sigma_{\infty}$ such that

$$
\lim _{n \rightarrow \infty} S_{i_{1} i_{2} \ldots i_{n}}\left(x_{0}\right)=x \text {. }
$$

Let $\mathbf{u}_{0}=\varnothing$, and for each $k>0$, there exists a unique $n_{k}$ such that $r_{i_{1} \ldots i_{n_{k}}} \leq$ $r^{k}<r_{i_{1} \ldots i_{n_{k}-1}}$, we let $\mathbf{u}_{k}=i_{1} i_{2} \ldots i_{n_{k}}$ and $\xi=\pi\left[\mathbf{u}_{0}, \mathbf{u}_{1}, \ldots\right]$. Then $\Phi(\xi)=x$ and $\Phi$ is surjective. To show that $\Phi$ is injective, we let $\xi=\pi\left[\mathbf{u}_{0}, \mathbf{u}_{1}, \ldots\right], \eta=$ $\pi\left[\mathbf{v}_{0}, \mathbf{v}_{1}, \ldots\right]$ and assume that

$$
\Phi(\xi)=\Phi(\eta)=x \in K,
$$

then $x \in K_{\mathbf{u}_{n}} \cap K_{\mathbf{v}_{n}}$ for all $n \geq 0$. Therefore $d\left(\mathbf{u}_{n}, \mathbf{v}_{n}\right) \leq 1$ and $\xi, \eta$ are equivalent.

Next we show that $\Phi$ is Hölder continuous on $\partial X$. Then being a bijective continuous map, $\Phi$ is a homeomorphism. Let

$$
\xi=\pi\left[\mathbf{u}_{0}, \mathbf{u}_{1}, \ldots\right] \text { and } \eta=\pi\left[\mathbf{v}_{0}, \mathbf{v}_{1}, \ldots\right]
$$

be any two non-equivalent geodesic rays in $X$. Then there exists a bilateral geodesic $\gamma$ joining $\xi$ and $\eta$ [24, p. 246]. By Proposition 2.2, we can assume that it is also canonical:

$$
\gamma=\pi\left[\ldots, \mathbf{u}_{n+1}, \mathbf{u}_{n}, \mathbf{t}_{1}, \mathbf{t}_{2}, \ldots, \mathbf{t}_{\ell}, \mathbf{v}_{n}, \mathbf{v}_{n+1}, \ldots\right]
$$


with $\mathbf{u}_{n}, \mathbf{t}_{1}, \ldots, \mathbf{t}_{\ell}, \mathbf{v}_{n} \in \mathcal{J}_{n}$. Fix an $x_{0} \in K$, it follows that (see the proof in Lemma 4.1)

$$
\left|S_{\mathbf{u}_{n}}\left(x_{0}\right)-S_{\mathbf{V}_{n}}\left(x_{0}\right)\right| \leq(\ell+2) r^{n} \operatorname{diam}(K),
$$

By Theorem 2.3, $\ell$ is uniformly bounded by a constant $k^{\prime}$, which depends on the graph only. Note that $\Phi(\xi) \in K_{\mathbf{u}_{k}}, \Phi(\eta) \in K_{\mathbf{v}_{k}}$ for all $k \geq 0$, hence

$$
\left|\Phi(\xi)-S_{\mathbf{u}_{n}}\left(x_{0}\right)\right|,\left|\Phi(\eta)-S_{\mathbf{v}_{n}}\left(x_{0}\right)\right| \leq r^{n} \operatorname{diam}(K) .
$$

This together with (4.2) implies that

$$
\begin{aligned}
& |\Phi(\xi)-\Phi(\eta)| \\
& \quad \leq\left|\Phi(\xi)-S_{\mathbf{u}_{n}}\left(x_{0}\right)\right|+\left|S_{\mathbf{u}_{n}}\left(x_{0}\right)-S_{\mathbf{v}_{n}}\left(x_{0}\right)\right|+\left|S_{\mathbf{v}_{n}}\left(x_{0}\right)-\Phi(\eta)\right| \\
& \quad \leq C^{\prime} r^{n},
\end{aligned}
$$

for some constant $C^{\prime}>0$. Since it is a bilateral canonical geodesic, we have $|\xi \wedge \eta|=n-(\ell+1) / 2$ and $\ell$ is uniformly bounded by a constant $k^{\prime}$. By using

$$
\rho_{a}(\xi, \eta)=\exp (-a|\xi \wedge \eta|)
$$

and (4.3), the theorem follows.

We can improve the map $\Phi$ to be Hölder equivalent under the following condition on the IFS:

(H) There exists $C^{\prime}>0$ such that for any integer $n>0$ and $\mathbf{v}, \mathbf{u} \in \mathcal{J}_{n}$, either

$$
S_{\mathbf{v}}(K) \cap S_{\mathbf{u}}(K) \neq \varnothing \quad \text { or } \quad\left|S_{\mathbf{v}}(x)-S_{\mathbf{u}}(y)\right| \geq C^{\prime} r^{n}
$$

for all $x, y \in K$.

Proposition 4.4. Suppose the IFS $\left\{S_{j}\right\}_{j=1}^{N}$ in Theorem 4.3 satisfies in addition condition $(\mathrm{H})$. Then there exists $C>0$ such that for any $\xi, \eta \in \partial X$,

$$
C^{-1}|\Phi(\xi)-\Phi(\eta)| \leq \rho_{a}(\xi, \eta)^{\alpha} \leq C|\Phi(\xi)-\Phi(\eta)|,
$$

where $\alpha=-\log r / a$.

Proof. We use the same notation as in the last theorem. Assume that $\xi \neq$ $\eta$. Since $\gamma$ in (4.1) is a geodesic, it follows that the $\mathbf{u}_{n+1} \nsim \mathbf{v}_{n+1}$, and hence $K_{\mathbf{v}_{n+1}} \cap K_{\mathbf{u}_{n+1}}=\varnothing$. Note that $\Phi(\xi) \in K_{\mathbf{v}_{n+1}}$ and $\Phi(\eta) \in K_{\mathbf{u}_{n+1}}$, condition (H) implies that

$$
|\Phi(\xi)-\Phi(\eta)| \geq C^{\prime} r^{n}
$$

This together with the estimation in Theorem 4.3 yields

$$
C^{\prime} r^{n} \leq|\Phi(\xi)-\Phi(\eta)| \leq C r^{n},
$$

and the theorem follows in view of the definition of hyperbolic metric $\rho_{a}$. 
There are important classes of IFS that satisfiy condition $(\mathrm{H})$. For example, we have the following result.

Proposition 4.5. Let $S_{i}(x)=A^{-1}\left(x+d_{i}\right), j=1, \ldots, N$ be contractive similitudes, where $A$ is a $d \times d$ integer matrix and $\left\{d_{1}, \ldots, d_{N}\right\} \subset \mathbb{Z}^{d}$. Then $\left\{S_{i}(x)\right\}_{i=1}^{N}$ satisfies condition $(\mathrm{H})$.

Proof. Let

$$
\alpha=\inf \left\{|x|: x \in \bigcup\left\{K-K+d: d \in \mathbb{Z}^{d} \text { s.t. } 0 \notin K-K+d\right\}\right\} .
$$

Since $K$ is compact, $\alpha>0$. For any $\mathbf{u} \in \Sigma_{n}$, we can write $S_{\mathbf{u}}(x)=A^{-n}\left(x+d_{\mathbf{u}}\right)=$ $r^{n} R^{n}\left(x+d_{\mathbf{u}}\right)$, where $0<r<1, d_{\mathbf{u}} \in \mathbb{Z}^{d}$ and $R$ orthonormal matrix. Suppose $S_{\mathbf{u}}(K) \cap S_{\mathbf{v}}(K)=\varnothing$, then for any $x, y \in K$, we have

$$
\left|S_{\mathbf{u}}(x)-S_{\mathbf{v}}(y)\right|=r^{n}\left|x-y+d_{\mathbf{u}}-d_{\mathbf{v}}\right|>0 .
$$

Noting that $d_{\mathbf{u}}, d_{\mathbf{v}} \in \mathbb{Z}^{d}$ and by making use of the expression $\alpha$ in (4.5), we have

$$
\left|S_{\mathbf{u}}(x)-S_{\mathbf{v}}(y)\right| \geq \alpha r^{n} .
$$

Condition $(\mathrm{H})$ is however not satisfied by an arbitrary IFS of similitudes with the OSC, hence we do not know if $\Phi$ in Theorem 4.3 can be improved to Hölder equivalence in general. We conclude this section by giving an example where condition $(\mathrm{H})$ fails.

Example 4.6. Let $A=\left[\begin{array}{ll}3 & 0 \\ 0 & 3\end{array}\right]$ and let $\mathcal{D}$ be the digit set consisting of the following vectors:

$$
\begin{array}{ll}
d_{1}=\left[\begin{array}{r}
-1 \\
0
\end{array}\right], \quad d_{2}=\left[\begin{array}{l}
0 \\
0
\end{array}\right], \quad d_{3}=\left[\begin{array}{l}
1 \\
0
\end{array}\right], \quad d_{4}=\left[\begin{array}{r}
-1 \\
1
\end{array}\right], \quad d_{5}=\left[\begin{array}{l}
0 \\
1
\end{array}\right], \\
d_{6}=\left[\begin{array}{l}
1 \\
1
\end{array}\right], & d_{7}=\left[\begin{array}{c}
-1+\eta \\
-1
\end{array}\right], \quad d_{8}=\left[\begin{array}{r}
\eta \\
-1
\end{array}\right], \quad d_{9}=\left[\begin{array}{c}
1+\eta \\
-1
\end{array}\right],
\end{array}
$$

where $\eta$ is a real number. The corresponding IFS is $S_{i}(x)=A^{-1}\left(x+d_{i}\right), i=$ $1, \ldots, 9$.

The example was used by Kenyon [17] (see also [20]) to demonstrate that the attractor $K$ is a self-similar tile and the tiling set $\mathcal{T}$ fails to be locally finite (in the sense of [17]). Indeed they showed that the relative position of two adjacent horizontal tiling sets are irrationally shifted relative to each other, and this shift can be made arbitrary small for a special choice of $\eta($ due to $m \eta(\bmod 1), m \in \mathbb{Z})$. Hence by using self-similarity in the microscopic scale, we see that condition $(\mathrm{H})$ is not satisfied. To be more concrete, we prove this and the OSC directly without recourse to the theory of tiles. 


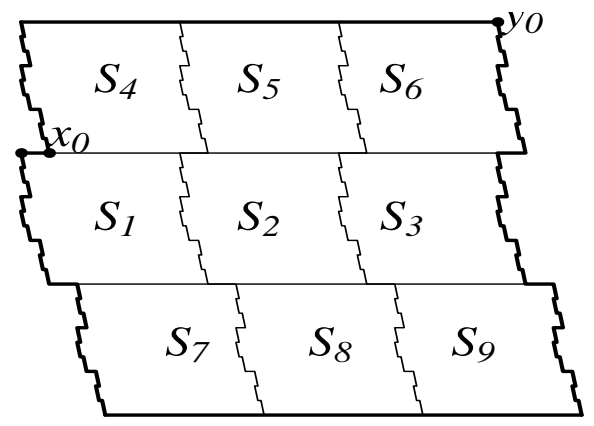

FIGURE 4.1. An example where condition $(\mathrm{H})$ fails

Let

$$
\begin{aligned}
& x_{0}=\lim _{n \rightarrow \infty} S_{47^{n}}(0)=\left(-\frac{1}{2}+\frac{\eta}{6}, \frac{1}{6}\right)^{t} ; \\
& y_{0}=\lim _{n \rightarrow \infty} S_{6^{n}}(0)=\left(\frac{1}{2}, \frac{1}{2}\right)^{t} .
\end{aligned}
$$

(See Figure 4.1 for the location and the corresponding indices for the points; it is seen from $x_{0}$ and $z_{0}=\lim _{n \rightarrow \infty} S_{14^{n}}(0)=\left(-\frac{1}{2}, \frac{1}{6}\right)^{t}$ that the relative shift of $S_{1}(K)$ and $S_{4}(K)$ is $\eta / 6$.) We take a special $\eta$ as follows. Let $a_{n} \in\{0,1\}, n=1,2, \ldots$ be such that $a_{1} a_{2} \ldots=010^{2} 10^{3} 10^{4} \ldots$. If we let $n_{k}$ to be the index of the $a_{n}$ that the $k$-th 1 appears, then

$$
n_{k}=(1+1)+(2+1)+\cdots+(k+1)=k(k+3) / 2 .
$$

Let $\eta / 6=\sum_{n=1}^{\infty} a_{n} 3^{-n}$. For each $k>0$, choose indexes $i_{1}, i_{2}, \ldots, i_{n_{k}} \in\{4,5,6\}$ such that

$$
d_{i_{j}}^{(1)}=a_{j}-1, j=1, \ldots, n_{k}-1, \quad \text { and } \quad d_{i_{n_{k}}}^{(1)}=a_{n_{k}}-2=-1 .
$$

We claim that this $S_{i_{1} \ldots i_{n_{k}}}(K)$ do not intersect $S_{47^{n_{k}-1}}(K)$. Indeed we consider the first coordinate of $x_{0}-S_{i_{1} \ldots i_{n_{k}}}\left(y_{0}\right)$; it is straightforward to check that

$$
\begin{aligned}
\rho_{n} & =\left(-\frac{1}{2}+\frac{\eta}{6}\right)-\left(\frac{d_{i_{1}}^{(1)}}{3}+\cdots+\frac{d_{i_{n_{k}}}^{(1)}}{3^{n_{k}}}+\frac{1}{2 \cdot 3^{n_{k}}}\right) \\
& =\frac{\eta}{6}-\left(\frac{1+d_{i_{1}}^{(1)}}{3}+\cdots+\frac{1+d_{i_{n_{k}}-1}^{(1)}}{3^{n_{k}-1}}+\frac{2+d_{i_{n_{k}}}^{(1)}}{3^{n_{k}}}\right)>0 .
\end{aligned}
$$


This implies that horizontally, $S_{i_{1} \ldots i_{n_{k}}}(K)$ is on the left side of $S_{47^{n_{k}-1}}(K)$ and hence they do not intersect. Therefore we have

$$
0<\operatorname{dist}\left(S_{i_{1} \ldots i_{n_{k}}}(K), S_{47^{n-1}}(K)\right) \leq \rho_{n_{k}}=\sum_{i=n_{k}+1}^{\infty} a_{i} 3^{-i}<3^{-\left(n_{k}+k\right)} .
$$

In view of (4.6), we see that the IFS does not satisfy $(\mathrm{H})$.

Next we show that the example satisfies the following equivalent condition of OSC [2] (which is of interest in the hyperbolic setup as it has the favor of group action): $\left\{S_{j}\right\}_{j=1}^{N}$ satisfies the OSC if and only if the identity map I is not in the closure of

$$
\subseteq=\left\{S_{\mathbf{u}}^{-1} \circ S_{\mathbf{v}} \mid \mathbf{u}, \mathbf{v} \in \Sigma_{n}, \mathbf{u} \neq \mathbf{v}, n>0\right\} .
$$

For any $\mathbf{u}=i_{1} \ldots i_{n}, \mathbf{v}=j_{1} \ldots j_{n} \in \Sigma_{n}=J_{n}$, with $i_{1} \neq j_{1}$, a direct calculation shows that

$$
\begin{aligned}
S_{\mathbf{u}}^{-1} \circ S_{\mathbf{v}}(x) & =x+\left(d_{j_{n}}-d_{i_{n}}\right)+A\left(d_{j_{n-1}}-d_{i_{n-1}}\right)+\cdots+A^{n-1}\left(d_{j_{1}}-d_{i_{1}}\right) \\
& :=x+\left(d^{(1)}, d^{(2)}\right)^{t} .
\end{aligned}
$$

It is straightforward to check that the second coordinate satisfies

$$
d^{(2)}=\left(d_{j_{n}}^{(2)}-d_{i_{n}}^{(2)}\right)+3\left(d_{j_{n-1}}^{(2)}-d_{i_{n-1}}^{(2)}\right)+\cdots+3^{n-1}\left(d_{j_{1}}^{(2)}-d_{i_{1}}^{(2)}\right) .
$$

Being integers, either (i) $d^{(2)}=0$ or (ii) $\left|d^{(2)}\right| \geq 1$. In (ii), $\left|S_{\mathbf{u}}^{-1} \circ S_{\mathbf{v}}(0)\right| \geq 1$ obviously. In (i) it is straightforward to check that $d_{j_{k}}^{(2)}-d_{i_{k}}^{(2)}=0$ for all $k=$ $1, \ldots, n$, and hence the indices satisfy $j_{k}, i_{k} \in r+\{1,2,3\}$ for $r=0,3$, 6. It follows that on the first coordinates, all $d_{j_{k}}^{(1)}-d_{i_{k}}^{(1)}$ are integers. By using the above argument to

$$
d^{(1)}=\left(d_{j_{n}}^{(1)}-d_{i_{n}}^{(1)}\right)+3\left(d_{j_{n-1}}^{(1)}-d_{i_{n-1}}^{(1)}\right)+\cdots+3^{n-1}\left(d_{j_{1}}^{(1)}-d_{i_{1}}^{(1)}\right),
$$

with the last term $3^{n-1}\left(d_{j_{1}}^{(1)}-d_{i_{1}}^{(1)}\right) \neq 0\left(\right.$ as $\left.i_{1} \neq j_{1}\right)$ and dominates the sum of the previous terms. We conclude that $d^{(1)}$ is a non-zero integer, so that $\left|S_{\mathbf{u}}^{-1} \circ S_{\mathbf{v}}(0)\right| \geq$ 1 also. Hence the identity map $I$ is not in the closure of $\subseteq$ and the IFS satisfies the OSC.

\section{REMARKS}

The previous consideration can be extended to certain self-affine sets by using the technique in [11] of replacing the Euclidean distance with a translational invariant "ultra-metric" adapted to the self-affine maps. 
Let $\left\{S_{j}\right\}_{j=1}^{N}$ be a set of self-affine maps on $\mathbb{R}^{d}$,

$$
S_{j}(x)=A^{-1}\left(x+d_{j}\right), \quad j=1, \ldots, N,
$$

where $d_{j} \in \mathbb{R}^{d}$ and $A$ is a $d \times d$ expanding matrix, i.e., all the eigenvalues have moduli $>1$. For $0<\varepsilon<\frac{1}{2}$, we choose a positive $C^{\infty}$ function $\varphi_{\varepsilon}(x)$ with support in the ball $B(0, \varepsilon)$ such that $\varphi_{\varepsilon}(-x)=\varphi_{\varepsilon}(x)$ and $\int \varphi_{\varepsilon}(x) \mathrm{d} x=1$. Let $V=A B(0,1) \backslash B(0,1)$, then $V$ is an annular region. Let $h(x)=\chi_{V} * \varphi_{\varepsilon}(x)$ be the convolution of the indicator function $\chi_{V}$ and $\varphi_{\varepsilon}(x)$. The pseudo-norm is defined as

$$
w(x)=\sum_{n=-\infty}^{\infty} q^{-n / d} h\left(A^{n} x\right), \quad x \in \mathbb{R}^{d},
$$

where $q=|\operatorname{det}(A)|>1$, we list some basic properties of $w(x)$ :

(i) $w(x)=w(-x), w(x)=0$ if and only if $x=0$;

(ii) $w(A x)=q^{1 / d} w(x) \geq w(x)$ for all $x \in \mathbb{R}^{d}$;

(iii) there exists $\beta>0$ such that

$$
w(x+y) \leq \beta \max \{w(x), w(y)\}, \quad \forall x, y \in \mathbb{R}^{d} .
$$

The details can be found in [11] and the references there. The function $w(x)$ defines an ultra-metric by $d_{w}(x, y)=w(x-y)$. It follows that

$$
d_{w}\left(S_{j}(x), S_{j}(y)\right)=q^{-1 / d} d_{w}(x, y), \quad \forall x, y \in \mathbb{R}^{d} .
$$

Hence these self-affine maps $S_{j}$ act as similitudes with contraction ratio $q^{-1 / d}$ with respect to this new distance $d_{w}(\cdot, \cdot)$. On the tree $\Sigma$, we define the horizontal edges on each $\Sigma_{n}$ as in (3.2). By some obvious modifications with the $d_{w}(\cdot, \cdot)$ replacing the Euclidean distance, it can be shown that Lemma 3.1 and the other theorems and propositions in Sections 3 and 4 remain true. We omit the details.

Another possible extension of the previous consideration is to assume the $\left\{S_{j}\right\}_{j=1}^{N}$ of similitudes to satisfy the weak separation condition ([18], [19]) or the finite type condition [22] instead of the open set condition. Similar to Lemma 3.1, we have that, for any $D \subset \mathbb{R}^{d}$ with $\operatorname{diam}(D) \leq a r^{n}$,

$$
\#\left\{K_{\mathbf{u}} \mid \mathbf{u} \in \mathcal{J}_{n}, K_{\mathbf{u}} \cap D \neq \varnothing\right\}
$$

is uniformly bounded. However, in this case the $\mathbf{u}$ corresponding to each $K_{\mathbf{u}}$ is not unique, and the counting in the symbolic space need to be adjusted. We conjecture that the same conclusion should hold as is for the open set condition. Without any separation condition, we believe the present construction of augmented root tree might also yield a hyperbolic graph. 
Acknowledgements. The authors were supported in part by an HKRGC grant, and X.Y. Wang was also supported by a postdoctoral fellowship from CUHK.

The authors are grateful to Professor V. Kaimanovich for bringing his attention to this question. They also thank Drs. L.F. Cheung, Q.R. Deng and H.B. Ju for many valuable discussions.

\section{REFERENCES}

[1] A. ANCONA, Théorie du potentiel sur les graphes et les variétés, 1990. MR 1100282 (92g:31012) (French)

[2] C. BANDT and S. GRAF, Self-similar sets. VII. A characterization of self-similar fractals with positive Hausdorff measure, Proc. Amer. Math. Soc. 114 (1992), 995-1001, http://dx.doi.org/10.2307/2159618. MR 1100644 (93d:28014)

[3] M. Coornaert, T. Delzant, and A. Papadopoulos, Géométrie et théorie des groupes. Les groupes hyperboliques de Gromov, Lecture Notes in Mathematics, vol. 1441, Springer-Verlag, Berlin, 1990, ISBN 3-540-52977-2. MR 1075994 (92f:57003) (French, with an English summary)

[4] M. Coornaert and A. Papadopoulos, Symbolic Dynamics and Hyperbolic Groups, Lecture Notes in Mathematics, vol. 1539, Springer-Verlag, Berlin, 1993, ISBN 3-540-56499-3. MR 1222644 (94d:58054)

[5] Q.-R. DENG and K.-S. LAU, Open set condition and post-critically finite self-similar sets, Nonlinearity 21 (2008), 1227-1232, http://dx.doi.org/10.1088/0951-7715/21/6/004. MR 2422376

[6] M. DenKer and H. SATO, Sierpinski gasket as a Martin boundary. I. Martin kernels, Potential Anal. 14 (2001), 211-232, http://dx.doi.org/10.1023/A:1011232724842. MR 1822915 (2002f:60139)

[7] _ Sierpinski gasket as a Martin boundary. II. The intrinsic metric, Publ. Res. Inst. Math. Sci. 35 (1999), 769-794, http://dx.doi.org/10.2977/prims/1195143423. MR 1739300 (2002f:60140)

[8] _ Reflections on harmonic analysis of the Sierpinski gasket, Math. Nachr. 241 (2002), 32-55, http://dx.doi.org/10.1002/1522-2616(200207)241:1;32::AID-MANA32:3.0.CO;2-5. MR 1912376 (2003e:28016)

[9] K. FALCONER, Fractal Geometry. Mathematical Foundations and Applications, John Wiley \& Sons Ltd., Chichester, 1990, ISBN 0-471-92287-0. MR 1102677 (92j:28008)

[10] M. GROMOV, Hyperbolic groups (1987), 75-263. MR 919829 (89e:20070)

[11] X.-G. HE and K.-S. LAU, On a generalized dimension of self-affine fractals, Math. Nachr. 281 (2008), 1142-1158, http://dx.doi.org/10.1002/mana.200510666. MR 2427166

[12] J.E. Hutchinson, Fractals and self-similarity, Indiana Univ. Math. J. 30 (1981), 713-747, http://dx.doi.org/10.1512/iumj.1981.30.30055. MR 625600 (82h:49026)

[13] H.B. JU, K.S. LAU, and X.Y. WANG, Self-similar sets as Martin boundaries (preprint).

[14] V.A. KaImANOVICH, Random walks on Sierpiński graphs: Hyperbolicity and stochastic homogenization (2003), 145-183. MR 2091703 (2005h:28022)

[15] J. Kigami, Harmonic calculus on p.c.f. self-similar sets, Trans. Amer. Math. Soc. 335 (1993), 721-755, http://dx.doi.org/10.2307/2154402. MR 1076617 (93d:39008)

[16] Analysis on Fractals, Cambridge Tracts in Mathematics, vol. 143, Cambridge University Press, Cambridge, 2001, ISBN 0-521-79321-1. MR 1840042 (2002c:28015) 
[17] R. KenYON, Self-replicating tilings (1992), 239-263. MR 1185093 (94a:52043)

[18] K.-S. LAU and S.-M. NGAI, A generalized finite type condition for iterated function systems, Adv. Math. 208 (2007), 647-671, http://dx.doi.org/10.1016/j.aim.2006.03.007. MR 2304331 (2008m:28007)

[19] K.-S. LAU, S.-M. NGAI, and X.-Y. WANG, Separation conditions for conformal iterated function systems, Monatsh. Math. 156 (2009), 325-355, http://dx.doi.org/10.1007/s00605-008-0052-4. MR 2486602

[20] J.C. LAgarias and Y. WANG, Self-affine tiles in $\mathbb{R}^{n}$, Adv. Math. 121 (1996), 21-49, http://dx.doi.org/10.1006/aima.1996.0045. MR 1399601 (97d:52034)

[21] K.-S. LAU and X.-Y. WANG, Iterated function systems with a weak separation condition, Studia Math. 161 (2004), 249-268, http://dx.doi.org/10.4064/sm161-3-3. MR 2033017 (2004j:28015)

[22] S.-M. NGAI and Y. WANG, Hausdorff dimension of self-similar sets with overlaps, J. London Math. Soc. (2) 63 (2001), 655-672, http://dx.doi.org/10.1017/S0024610701001946. MR 1825981 (2002c:28010)

[23] A. SCHIEF, Separation properties for self-similar sets, Proc. Amer. Math. Soc. 122 (1994), $111-$ 115, http://dx.doi.org/10.2307/2160849. MR 1191872 (94k:28012)

[24] W. Woess, Random Walks on Infinite Graphs and Groups, Cambridge Tracts in Mathematics, vol. 138, Cambridge University Press, Cambridge, 2000, ISBN 0-521-55292-3. MR 1743100 (2001k:60006)

KA-SING LAU:

The Chinese University Of Hong Kong

Shatin, NT, Hong Kong, P.R. China.

E-MAIL: kslau@math.cuhk.edu.hk

XIANG-YANG WANG:

School of Mathematics and Computational Science

Zhong-Shan University

510275 Guang-Zhou. P.R. China.

E-MAIL: mcswxy@mail.sysu.edu.cn

KEY WORDS AND PHRASES: boundary, geodesic ray, hyperbolic graph, iterated function system, self-similar sets, open set condition.

2000 Mathematics SubJeCt Classification: 28A78 (28A80).

Received: April 15th, 2008; revised: October 2nd, 2008.

Article electronically published on July 31st, 2009. 